\title{
A Note on the Pay-off Matrix in Multiple Objective Programming
}

\section{INTRODUCTION}

ONE OF the key concepts in multiple objective optimization is the pay-off matrix. The elements of the payoff matrix $P$ are defined as: $p_{i j}=\mathbf{g}_{(}\left(\bar{x}^{\prime}\right), i, j=1, \ldots, m$, where $g_{i}(x), i=1, \ldots, m$ are the goal variables' to be minimized as functions of the instruments $x$ and $x$ denotes the instrument vector which minimizes the $i$ th goal variable within the feasible region $K$. The pay-off matrix is a valuable tool in investigating the conflicts between the goals of the decision problem at hand. Two important vectors which can be derived from this matrix are the ideal (utopia) vector $\mathbf{g}^{*}=\left(\mathrm{g}_{1}^{*}, \ldots, \mathrm{g}_{m}^{*}\right)$, with

$$
\mathbf{g}_{j}^{\star}=\mathbf{g}_{j}\left(\tilde{x}^{j}\right), j=1, \ldots, m,
$$

and the nadir vector $\mathbf{n}^{\star}=\left(\mathbf{n}_{1}^{*}, \ldots, \mathbf{n}_{m}^{\star}\right)$ with:

$$
\mathbf{n}_{j}^{\star}=\max _{i=1 \ldots, m} \mathbf{g}_{j}\left(\bar{x}^{i}\right), j=1, \ldots, m .
$$

For example, given the pay-off matrix:

\begin{tabular}{lccc} 
& $\bar{x}^{i}$ & $\bar{x}^{2}$ & \multicolumn{1}{c}{$\bar{x}^{3}$} \\
\cline { 2 - 4 } $\mathbf{g}_{1}$ & 7 & 9 & 8 \\
$\mathbf{g}_{2}$ & 9 & 3 & 15 \\
$\mathbf{g}_{3}$ & 6 & 4 & 1
\end{tabular}

the ideal vector $\left(\mathbf{g}^{*}\right)$ and nadir vector $\left(\mathbf{n}^{*}\right)$ are: $\mathbf{g}^{*}=(7,3,1)$ and $\mathbf{n}^{*}=(9,15,6)$.

Both the ideal and the nadir vectors are frequently used in interactive methods (e.g. in STEM-type methods). The pay-off matrix is not necessarily unique, because different $\bar{x}^{j}$ may produce the same $\mathbf{g}_{j}^{*}$ value, thus the nadir vector is not necessarily unique either. In this paper we address this problem by first presenting two examples with non-unique nadir vectors (Section 2). Next, we re(de)fine the concept of the nadir vector (Section 3). A procedure for determining the nadir vector is given in Section 4. Finally, we discuss the computational complexity of the proposed procedure.

\section{NON-UNIQUE PAY-OFF MATRICES}

The possible implications of a non-unique pay-off matrix for the calculation of the nadir vector can best be demonstrated intuitively by two simple examples. In both cases there is a set of possible nadir vectors, only one of which is the 'true' one. The first example shows that a randomly chosen nadir vector may have higher values than the true nadir vector. In the second example we show that a randomly chosen nadir vector may have lower values than the true nadir vector.

\section{Example I}

In Fig. 1, the set of feasible solutions of a given multiple objective programming problem is represented

'In the literature, one often finds the term objective function instead of goal variable. Our preference for the term goal variable is explained in [3].

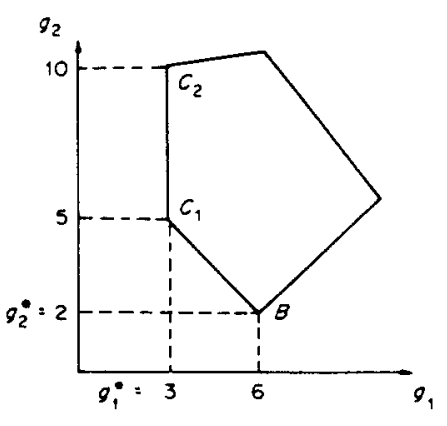

Fig. 1.

in goal value space. Clearly, there is only one solution $(B)$ yielding the optimal goal value $g_{2}^{*}$. In contrast, all solutions on the line segment $\left(C_{1}, C_{2}\right)$ are optimal with respect to $g_{1}(x)$. If no attention were paid to the nonuniqueness of the optimum for goal variables $g_{1}(x)$ different pay-off matrices and different nadir vectors might result. In this case, we might have as pay-off matrix either

$$
P_{1}=\left(\begin{array}{ll}
3 & 6 \\
5 & 2
\end{array}\right) \text { or } P_{2}=\left(\begin{array}{rr}
3 & 6 \\
10 & 2
\end{array}\right)
$$

with corresponding nadir vectors

$$
\mathbf{n}_{1}^{*}=(6,5) \text { or } \mathbf{n}_{2}^{*}=(6,10)
$$

The fact that $C_{1}$ dominates all alternative solutions on the line segment $\left(C_{1}, C_{2}\right)$ is an intuitive reason for defining $n_{1}^{*}$ as the 'true' nadir vector. In other words, if the nadir values were imposed as constraints (e.g. in STEM-type methods), the $n_{1}^{*}$-values would exclude a larger number of inferior (dominated) solutions from the remaining set of feasible solutions than all other possible nadir vectors, At the same time, the $\mathbf{n}_{1}^{*}$-values would not exclude any efficient solution.

\section{Example 2}

The second example is a problem described in [2]. In this problem, each of three goal variables is to be minimized. The optimum of the first goal variable is unique whereas the optimum of both other goal variables is non-unique. In Table 1 we summarize the alternative goal vectors (corner solutions) yielding the optimal goal values.

Table 1 .

\begin{tabular}{lcrcccccc}
\hline & $\mathbf{g}_{1}^{*}$ & \multicolumn{6}{c}{$\mathbf{g}_{3}^{*}$} \\
\hline $\mathbf{g}_{1}$ & 28.75 & 30 & 30.5 & 29.375 & 32.75 & 28.75 & 32.5 \\
$\mathbf{g}_{2}$ & 250 & 5 & 5 & 225.5 & 120.5 & 250 & 133.3 \\
$\mathbf{g}_{3}$ & 0 & 50 & 45 & 0 & 0 & 0 & 0 \\
\hline
\end{tabular}

Using these solutions, eight different pay-off matrices can be constructed, resulting in six different nadir vectors. Apart from the goal vector $(32.5,133.3,0)$, all alternative solutions are efficient. The 'true' nadir vector would (intuitively) be defined as the vector of maximum values obtained within the given set of efficient goal 
vectors. That is, the true nadir vector would be

$$
\mathbf{n}_{T}^{*}=(32.75,250,50)
$$

Notice, that if any of the other goal vectors were selected as nadir vectors and if the values of such a nadir vector were imposed as constraints, a subset of the efficient set would be excluded. For instance, the values of the potential nadir vector $(32.75,250,45)$ would, if imposed as constraints, exclude the goal vector $(30,5,50)$ which is non the less an efficient solution.

\section{CELLAR, NADIR AND PESSIMISTIC VECTORS}

To solve the problems sketched above, we need a more precise definition of the nadir vector concept. Let us denote the feasible set of the decision problem by $K$ and the efficient set by $E$. In addition, define $K_{i}=\{x \mid x \in K$ and $\mathbf{g}_{1}(x)=\mathbf{g}_{t}^{*}$, with

$$
\mathrm{g}_{i}^{\star}=\min _{x \in K} \mathbf{g}_{i}(x)
$$

Furthermore, let

$$
K_{\mu_{i}}=\left\{x \mid x \in K_{i} \text { and } \mathbf{g}_{j}(x)=\mathbf{g}_{, i}^{*}\right\}
$$

with

$$
\begin{aligned}
\mathbf{g}_{j i}^{*} & =\min _{x \in K_{1}} \mathbf{g}_{j}(x) ; \\
K_{k|j| i} & =\left\{x \mid x \in K_{j \mid i} \text { and } \mathbf{g}_{k}(x)=\mathbf{g}_{k \mid / j i}^{*}\right\} ;
\end{aligned}
$$

with

$$
\mathbf{g}_{* j \mid i}=\min _{x \in K_{j \mid i}} \mathbf{g}_{j}(x) \text {; etc. }
$$

Next, we define the nadir vector $\boldsymbol{n}^{*}$ as the vector of goal values of which the $j$ th element is given by

$$
\mathbf{n}_{j}^{*}=\max _{x \in K}\left\{\mathbf{g}_{j}(x)\right\}
$$

where

$$
K \text { is the union of all } K_{i|| \ldots \mid i_{m}} \text {, }
$$

with

$$
i_{k}=1, \ldots, m \text { for each } k=1, \ldots, m .
$$

The reader can easily verify that this nadir vector is unique. It is often assumed that the elements of the nadir vector represent the worst values of the goal variables over the entire efficient set. In many cases this is. not correct (not even with the precise definition of the nadir vector), as is shown in [1], [3] and [5]. Therefore, we define the cellar vector $\mathrm{c}$ as the vector whose elements are the worst values of the goal variables over the entire efficient set:

$$
\mathbf{c}_{j}=\max _{\boldsymbol{s} \in E}\left\{\mathbf{g}_{j}(x)\right\}, j=1, \ldots, m .
$$

As mentioned above, for the redefined nadir vector $\mathbf{n}_{j}^{*} \leq \mathbf{c}_{j}$ for $j=1, \ldots, m$ in the general vector $\min$. imization problem.

The elements of the nadir and cellar vector can be seen as parameters of the decision problem at hand, which are independent of the preferences of the decision-maker. The term pessimistic vector is used for the maximally required attainments determined by the decision maker. The elements of the pessimistic vector $p$ are the highest goal values which the decision-maker considers to be acceptable. Obviously, the decision-maker may choose any (feasible) value of $\mathbf{p}_{i}$. That is, he may choose $\mathbf{p}_{1} \geq \mathbf{c}_{1}, \mathbf{n}_{i}^{*} \leq \mathbf{p}_{j} \leq \mathbf{c}_{i}$, or $\mathbf{p}_{1} \leq \mathbf{n}_{j}^{*}$. If the decision-maker does not know exactly what he wants. one should be careful in defining the $p$-values for him. In well-defined models, it is reasonable to choose $\mathbf{p}_{j}=\mathbf{c}_{j}, j=1, \ldots, m$.

\section{DETERMINATION OF THE NADIR VECTOR}

The definition of the nadir vector suggests that it is necessary to solve a great number of optimization problems. However, if the solution of one of these problems-e.g.

$$
\min _{x \in K} \mathbf{g}_{j}(x) \text {-is unique, }
$$

then it is immediately clear that this solution is the only feasible vector in $\mathbf{K}_{i 1}\left|\ldots i_{m-1}\right| j, i_{1} \neq j, \ldots i_{m-1} \neq j$. This means that testing whether the solution of the optimization problem at hand is unique or not, may reduce the number of optimization problems considerably.

To avoid unnecessary technical details. we will not give a formal presentation of the procedure. Instead, we illustrate it by means of a simple decision problem with three goal variables $g_{1}(x), g_{2}(x)$ and $g_{3}(x)$. The elaboration of the procedure is summarized in Fig. 2. First, we set all elements of the nadir vector equal to infinity. Then, the first goal variable, $g_{1}(x)$, is optimized within the set of alternatives, $K$, and we test whether the solution of this optimization problem is unique. In this example, the solution is unique. The nadir vector is updated by taking as its elements the values of the goal variables in this unique solution. Then the goal variable $\mathbf{g}_{2}(x)$ is optimized. Now we have alternative solutions, by which it becomes necessary to perform another two optimizations. From the flow-chart in Fig. 2 it can be concluded that the solution of

$$
\min _{x \in K_{2}} \mathbf{g}_{1}(x)
$$

is unique. Therefore, the nadir vector must be updated by adopting those goal values resulting from

$$
\min _{x \in K_{2}} \mathbf{g}_{1}(x)
$$

which are higher than the corresponding values in the old nadir vector. The solution of

$$
\min _{x \in K_{2}} \mathbf{g}_{3}(x)
$$

is not unique, so we have to perform another optimization:

$$
\min _{x \in K_{3 ; 2}} g_{1}(x), \text { to. }
$$

Finally, we add a technical remark concerning the implementation of the procedure. Testing whether there are alternative solutions or not can be carried out with the non-basic reduced cost vector: if at least one element of this vector related with the non-basic variables in the final row of the Dantzig simplex tableau (see [4]) is the zero coefficient, we have alternative optima. There exists no other procedure which calculates the nadir vector as defined above. However, alternative procedures can be proposed which guarantee that $\mathbf{n}^{*} \leq \mathbf{c}_{j}, j=1, \ldots, m$. Among others, one can determine such a nadir vector by performing the optimization problems:

$$
\min \left\{\mathbf{g}_{i}(x)+\sum_{j \neq 1} \epsilon \mathbf{g}_{j}(x)\right\}, i=1, \ldots, m
$$




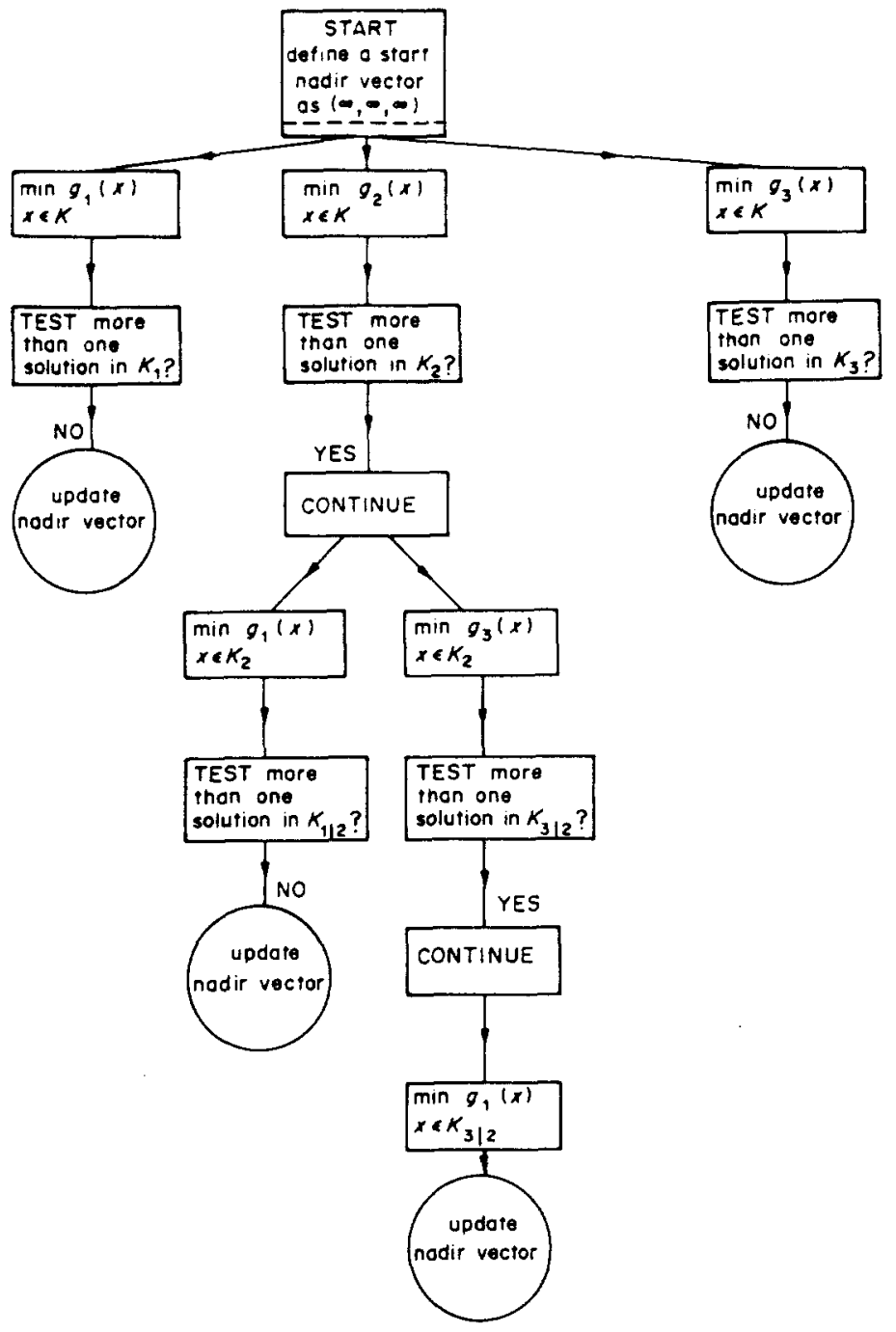

Fig. 2.

with $\epsilon$ a small positive scalar, or lexmin

$$
\left\{\mathbf{g}_{i}(x), \mathbf{g}_{i+1}(x), \ldots, \mathbf{g}_{1}(\mathbf{x}), \ldots, \mathbf{g}_{i-1}(x)\right\}, i=1, \ldots, m \text {. }
$$

\section{DISCUSSION}

It can easily be verified that the structure of the proposed procedure is a tree, in which the nodes correspond to the optimization problems. If there are $m$ goal variables, the maximum number of nodes $\alpha(m)$ is given by

$$
\alpha(m)=\sum_{p=0}^{m-1} \frac{m !}{\mathbf{p} !} .
$$

Of course, the formula given above represents the worst case where all optimization problems in the procedure have alternative optima. In general, only some of the optimization problems will have alternative optima, limiting the number of problems to be optimized. Second, as mentioned above, the redefined nadir vector is not equal to the cellar vector (Section 3). Nevertheless, the nadir vector as defined here may constitute a good starting point for finding the elements of the cellar vector. Of course, other starting points might be appropriate. However, the question which is the best one cannot be answered before a good procedure to find the cellar vector is available.

\section{ACKNOWLEDGEMENT}

The authors like to thank FA Lootsma (Delft University of Technology), W Ogryczak (University of Warsaw) and an anonymous referee for their valuable remarks.

\section{REFERENCES}

1. Dessouki MI, Ghiassi $M$ and Davis WJ (1979) Determining the worst value of an objective function within the nondominated solutions in multiple objective linear programming. University of Illinois, Urbana. 
2. Kok M (1984) The interface with decision makers in interactive multi-objective linear programming. Report 84-38. Dep. of Math. and Inf., Delft.

3. Spronk J (1981) Interactive Multiple Goal Programming: Applications to Financial Planning. Martinus Nijhof, Boston.

4. Wagner HM (1975) Principles of Operations Research, with Applications to Managerial Decisions. Prentice-Hall, London.

5. Weistroffer HR (1983) On pessimitic values in multiple objectives optimization. National Research Institute for Math. Sc. I482, Pretoria.

Matthijs Kok

Jaap Spronk ${ }^{2}$

(Augusi 1985)

'Unizersity of Technology

P.O. Box 356

2600 AJ Delft

The Netherlands

'Erasmus Unicersity

P.O. Bo. 1738

3000 DR Rotterdam

The Netherlands 